\title{
Effect of thyrotropin suppressive therapy on lumbar bone mineral density in patients with differentiated thyroid cancer: a retrospective cohort study
}

\author{
Xinshui Wang ${ }^{1}$, Ruoling Teng ${ }^{2}$, Fenfen Liu ${ }^{2}$, Ping Liu ${ }^{2}$, Yujiao Yang ${ }^{2}$ \\ ${ }^{1}$ Department of Endocrinology, the Third Affiliated Hospital of Soochow University, Changzhou, China; ${ }^{2}$ Department of Geriatrics, the Third \\ Affiliated Hospital of Soochow University, Changzhou, China \\ Contributions: (I) Conception and design: All authors; (II) Administrative support: Y Yang; (III) Provision of study materials or patients: X Wang, \\ Y Yang; (IV) Collection and assembly of data: X Wang, R Teng, F Liu, P Liu; (V) Data analysis and interpretation: X Wang, R Teng, Y Yang; (VI) \\ Manuscript writing: All authors; (VII) Final approval of manuscript: All authors. \\ Correspondence to: Yujiao Yang. Department of Geriatrics, the Third Affiliated Hospital of Soochow University, No. 185, Juqian Street, Changzhou \\ 213003, China. Email: yangyujiao@czfph.com.
}

\begin{abstract}
Background To investigate the effect of thyrotropin (TSH) suppressive therapy on lumbar bone mineral density (BMD) in patients with differentiated thyroid cancer (DTC) after operation.

Methods: We retrospectively analyzed 70 DTC patients at intermediate or high risk of recurrence, including 17 males, 30 premenopausal females, and 23 postmenopausal females. All patients were treated with oral $131 \mathrm{I}$ to clear any residual thyroid and L-thyroxine tablets to suppress TSH after surgery. The baseline and follow-up lumbar BMD were measured. The anthropometric and biochemical parameters, the doses of calcium supplement, and levothyroxine were collected.

Results: Lumbar BMD in the postmenopausal female group was markedly decreased (regression coefficient: $-0.201 ; \mathrm{P}<0.001$ ) compared to the male group and premenopausal female group (both: $\mathrm{P}>0.05$ ). Further comparisons between groups found that premenopausal women had a monthly lumbar BMD reduction of $0.001 \mathrm{~g} / \mathrm{cm}^{2}$ more than men, but the difference was not statistically significant $(\mathrm{P}=0.515)$. In contrast, postmenopausal women had a monthly lumbar BMD reduction of $0.004 \mathrm{~g} / \mathrm{cm}^{2}$ more than men and $0.003 \mathrm{~g} / \mathrm{cm}^{2}$ more than postmenopausal controls $(\mathrm{P}=0.017$ and $\mathrm{P}<0.001$, respectively). Lumbar $\mathrm{BMD}$ decreased significantly with the increasing duration of TSH suppression in both the calcium supplement group and the non-calcium supplement group (both: $\mathrm{P}<0.05$ ), but there was no statistical difference between the two groups $(\mathrm{P}=0.534)$.

Conclusions: The longer the duration of TSH suppression in DTC patients after operation, the more significant the decrease of BMD, especially in postmenopausal women. Furthermore, calcium supplementation did not significantly improve lumbar BMD.
\end{abstract}

Keywords: Thyroid neoplasms; thyrotropin (TSH); bone density; postmenopause

Submitted Dec 22, 2021. Accepted for publication Feb 18, 2022.

doi: $10.21037 / \mathrm{gs}-22-50$

View this article at: https://dx.doi.org/10.21037/gs-22-50

\section{Introduction}

Thyroid cancer is the most common endocrine malignant tumor, and differentiated thyroid cancer (DTC) is the most prevalent type of thyroid cancer, which can account for over $90 \%$ of all thyroid cancers (1-3). The management of DTC includes risk assessment, surgical management, radioiodine (RAI) remnant ablation and therapy, and thyrotropin (TSH) suppression therapy using levothyroxine $(4,5)$. After ${ }^{131} \mathrm{I}$ treatment following surgery, TSH suppressive therapy should be given to the patients promptly to reduce the 
recurrence rate of DTC. The therapeutic value of TSH suppressive therapy for DTC has been proved $(4,6,7)$.

It is well known that the metabolism, development, and reconstruction of bone are affected by the thyroid hormone. It was reported that lower TSH levels, even within the normal range, negatively affect the BMD (8). Furthermore, thyroid dysfunction has been known to represent a risk factor for bone disease $(9,10)$. Both hyperthyroidism and subclinical hyperthyroidism were reported to affect bone metabolism resulting in decreased bone mineral density (BMD) and increased risk of fracture (10). TSH suppressive therapy leads to changes in the internal environment of patients, and sustained subclinical hyperthyroidism interferes with bone metabolism (11).

There are many studies on the effect of TSH suppressive therapy on BMD of patients with DTC, but the conclusions are divergent (12-22). Multiple studies have shown that TSH suppressive therapy can increase the risk of osteoporosis in patients with DTC (13-17). At the same time, many studies have demonstrated that TSH suppressive therapy does not reduce the BMD of the lumbar spine in patients with DTC (18-22). However, in some of the above studies, only female DTC patients or only female patients were included in the BMD test $(13,15-19,22)$. Only two trials studied the effect of a calcium supplement or vitamin D supplement on BMD in DTC patients $(14,17)$. A meta-analysis by Yoon et al. [2019] confirmed that, in postmenopausal women with DTC (instead of premenopausal women and men), there might be a link between chronic TSH suppression therapy and lower BMD in the spine and total hip (23). However, a metaanalysis by Wang et al. [2020] suggested that there was no significant difference in the lumbar BMD between the patients with DTC after TSH-suppressive therapy and the control groups (24). These two meta-analyses produced inconsistent results. Additionally, these results need to be interpreted with caution. Heterogeneity was observed in the above two meta-analyses results, and the results did not take into account the interactions among age, body mass index (BMI), and calcium supplementation.

Therefore, we designed this retrospective study to evaluate the effect of TSH suppressive therapy on lumbar BMD in DTC patients after surgery and RAI therapy, and to analyze whether this effect has a population difference. Another objective of this study was to evaluate whether calcium supplements can help improve BMD. We present the following article in accordance with the STROBE reporting checklist (available at https://gs.amegroups.com/ article/view/10.21037/gs-22-50/rc).

\section{Methods}

\section{Patients}

This was a retrospective cohort study. Patients who were scheduled to receive ${ }^{131} \mathrm{I}$ treatment to remove residual thyroid tissue or as part of routine follow-up after DTC operation in our nuclear medicine discipline between October 2013 and September 2019 were eligible for the study. All patients took ${ }^{131} \mathrm{I}$ solution after the surgery for thyroid ablation. After treatment, the patients took levothyroxine tablets to suppress TSH to an initial target level. For DTC patients at high-risk of recurrence, it is recommended by the American Thyroid Association (ATA) guidelines to suppress the initial TSH to below $0.1 \mathrm{mIU} / \mathrm{L}$ $(2,25)$. For DTC patients at intermediate-risk of recurrence, it is recommended by the ATA guidelines to suppress the initial TSH to $0.1-0.5 \mathrm{mIU} / \mathrm{L}(2,25)$. All the included patients met the following criteria: (I) intermediate or high risk of recurrence after total or subtotal removal of DTC; (II) good treatment compliance, regular administration of levothyroxine in a timely manner, and achieved the initial TSH target during the initial treatment (the first year); and (III) the baseline BMD was measured after surgery, and the patients were followed up regularly to receive the BMD tests. The risk stratification after surgery was done according to the 2009 ATA guidelines. Intermediate-risk of recurrence met one of the following criteria: (I) microscopic invasion of the tumor into the perithyroidal soft tissues at initial surgery; (II) cervical lymph node metastases or ${ }^{131} \mathrm{I}$ uptake outside the thyroid bed on the whole-body RAI scan done after thyroid remnant ablation; or (III) a tumor with aggressive histology or vascular invasion. High-risk of recurrence met one of the following criteria: (I) macroscopic tumor invasion; (II) incomplete tumor resection; (III) distant metastases; or (IV) thyroglobulinemia out of proportion to what is seen on the posttreatment scan (25).

A patient was excluded if they had or were any of the following: (I) hypoparathyroidism, serum level of parathyroid hormone (PTH) $<16 \mathrm{pg} / \mathrm{mL}$; (II) hypocalcemia, serum level of calcium $<2.11 \mathrm{mmol} / \mathrm{L}$; (III) bone metastasis; (IV) taking steroids; (V) treated with medications for osteoporosis; (VI) taking steroid hormones or steroid-modulating medications or (VII) missing or incomplete data.

This study was conducted following the principles of the Declaration of Helsinki (as revised in 2013) and was approved 
by the ethics committee of the Third Affiliated Hospital of Soochow University [approval No. (2020) WD-018]. Written informed consent was obtained from the patients.

\section{Study methods}

The anthropometric and biochemical parameters, the dose of calcium supplement and L-thyroxine, and the lumbar BMD score of the included patients were collected. Anthropometric parameters such as height and weight were measured while the patients were dressed in lightweight clothing. BMI was calculated using the following formula: weight in kilograms divided by the square of the height in meters $\left(\mathrm{kg} / \mathrm{m}^{2}\right)$.

The concentrations of serum calcium and serum 25-hydroxyvitamin D were measured using an automated biochemical analyzer (Beckman Coulter AU5831, USA). The serum level of PTH was measured based on a chemiluminescence immunoassay (Siemens ADVIA Centaur, Germany). Concentrations of serum free T3 (FT3), serum free T4 (FT4), and TSH were evaluated with the radioimmunoassay method (Cobas 8000 , Switzerland).

BMD was determined by DXA (dual-energy X-ray absorptiometry, Hologic, Discovery, WI, USA) and was performed on the lumbar spine (L1-L4) area. All DXA scans were performed by trained and certified DXA technicians. The BMD values were measured in $\mathrm{g} / \mathrm{cm}^{2}$. $\mathrm{BMD}$ of the lumbar spine was measured at the time of initial ${ }^{131} \mathrm{I}$ admission (baseline) and during the follow-up period. If there were multiple BMD follow-ups during the TSH suppression period, the data from the final follow up was used for the statistical analysis.

The postmenopausal female controls $(n=21)$ were randomly selected among participants that had more than two health check-up records, including BMD test, in the Third Affiliated Hospital of Soochow University. Participants who had any history of thyroid disease, diabetes, kidney disease, heart failure, or any other major medical conditions were excluded. All of the postmenopausal female controls had not taken any medications known to affect bone metabolism except calcium supplements. We also collected data on the BMI, serum levels of calcium, PTH, FT3, FT4, $\mathrm{TSH}$, and BMD score of the postmenopausal controls.

\section{Statistical analysis}

Continuous variables were expressed as mean $\pm \mathrm{SD}$ or median (Q1-Q3), while categorical variables were expressed as frequency (\%). Non-paired Student's $t$-test or Mann-Whitney nonparametric test was used to compare continuous variables. Pearson chi-square test and Fisher exact test were used to analyze categorical variables.

We analyzed the trend of lumbar BMD after TSH suppression using linear regression. Afterwards, we used the generalized additive mixed model (GAMM) to examine the changes of BMD with the duration of TSH suppression or the duration of follow-up in different population groups and different calcium supplement groups. GAMM eliminated the influence of individual differences on the results of repeated measurements by introducing random effects into the statistical analysis (26). The linear relationship between lumbar BMD change and duration of TSH suppression or duration of follow-up was determined. The results were then adjusted for potential confounding factors such as age, BMI, risk of recurrence, calcium supplement, the dose of L-thyroxine, and the serum level of TSH, FT3, FT4, PTH, calcium, and 25-hydroxyvitamin D. All analyses were performed by using $\mathrm{R}$, version 3.4.3 (https://www.r-project. org/). $\mathrm{P}<0.05$ was considered statistically significant, and all statistical tests were two-tailed.

\section{Results}

\section{Analysis of the baseline status of enrolled patients}

Initially, 144 patients with postoperative DTC were eligible for the study, among which 74 patients were excluded, including 66 patients who only had one BMD test, 4 patients with hypoparathyroidism, and 4 patients with hypocalcemia. Eventually, 70 patients were included in the study (Figure 1). The patients were divided into the male group (17 cases), the premenopausal female group (30 cases), and the postmenopausal female group (23 cases). There was no significant difference in BMI, risk of recurrence, the proportion of patients who used calcium supplements, the number of BMD tests, suppression time, serum level of TSH, FT3, FT4, PTH, calcium, and 25 -hydroxyvitamin $\mathrm{D}$ among the three groups $(\mathrm{P}>0.05)$. However, there was a significant difference in age, the dose of L-thyroxine, and BMD of the lumbar spine among the three groups $(\mathrm{P}<0.001 ;$ Table 1).

\section{Changes of lumbar BMD with a duration of TSH suppression in the general population}

After adjusting for age, BMI, risk of recurrence, calcium 


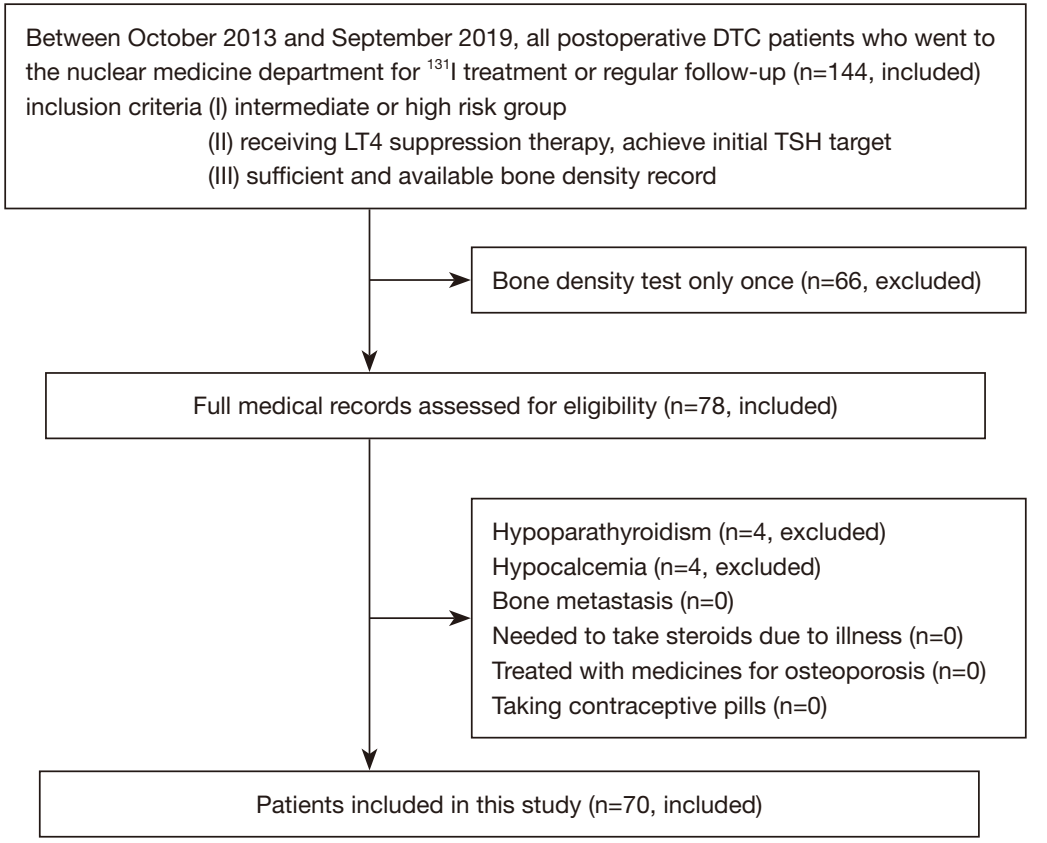

Figure 1 Flowchart of the study population. DTC, differentiated thyroid cancer; LT4, L-thyroxine; TSH, thyrotropin.

supplement, the dose of L-thyroxine, and the serum level of TSH, FT3, FT4, PTH, calcium, and 25-hydroxyvitamin $\mathrm{D}$, the overall BMD value of all patients in the study decreased significantly with the increasing duration of TSH suppression (regression coefficient: $-0.142 ; \mathrm{P}<0.001$ ) (Figure 2).

\section{Changes of lumbar BMD with suppression time in different population groups}

Changes of lumbar BMD with suppression time among the male, premenopausal. and postmenopausal female groups

After adjusting for age, BMI, risk of recurrence, calcium supplement, the dose of L-thyroxine, and the serum level of TSH, FT3, FT4, PTH, calcium, and 25-hydroxyvitamin $\mathrm{D}$, the changes of BMD of the lumbar spine in the male and premenopausal female groups were not significant (regression coefficient: 0.058 and -0.051 ; $\mathrm{P}$ value: 0.483 and 0.308 , respectively). However, the BMD of the lumbar spine in postmenopausal females showed a significant downward trend (regression coefficient: -0.201 ; $\mathrm{P}<0.001)$. We found that the decrease of lumbar BMD in the premenopausal female group was $0.001 \mathrm{~g} / \mathrm{cm}^{2}$ more than that of the male group per month, but there was no statistical significance $(\mathrm{P}=0.515)$. In contrast, the decrease of lumbar BMD in the postmenopausal female group was $0.004 \mathrm{~g} / \mathrm{cm}^{2}$ more than that of the male group per month, which was statistically significant $(\mathrm{P}=0.017)$. We further analyzed the interaction between different population groups and calcium supplementation. The results showed that there was no interaction between diverse populations and calcium supplementation $(\mathrm{P}>0.05)$ (Figure 3$)$.

\section{Changes of lumbar BMD with the duration of follow-up between postmenopausal patients and postmenopausal controls}

There was no significant difference in age, BMI, calcium supplement, serum level of PTH, calcium, and BMD of the lumbar spine between the two groups $(\mathrm{P}>0.05)$. However, there was a significant difference in the serum level of TSH, FT3, and FT4, and the duration of follow-up between the two groups $(\mathrm{P}<0.001$; Table 2). After adjusting for age, BMI, calcium supplement, and the serum level of TSH, FT3, FT4, PTH, and calcium, the changes of BMD of the lumbar spine in the postmenopausal controls were not significant (regression coefficient: 0.014; $\mathrm{P}=0.457$ ). However, the $\mathrm{BMD}$ of the lumbar spine in postmenopausal patients showed a significant downward trend (regression coefficient: $-0.365 ; \mathrm{P}<0.001)$. The decrease of lumbar BMD in the postmenopausal women patient group was $0.003 \mathrm{~g} / \mathrm{cm}^{2}$ more than that in the postmenopausal control group per 
Table 1 Comparison of general data among different populations

\begin{tabular}{|c|c|c|c|c|}
\hline Group & Male & Premenopausal female & Postmenopausal female & $P$ value \\
\hline Age (years) & $46.9 \pm 13.2$ & $42.1 \pm 8.4$ & $57.2 \pm 7.0$ & $<0.001$ \\
\hline $\mathrm{BMI}\left(\mathrm{kg} / \mathrm{m}^{2}\right)$ & $26.4 \pm 2.6$ & $24.0 \pm 3.0$ & $24.8 \pm 4.0$ & 0.055 \\
\hline Risk of recurrence & & & & 0.100 \\
\hline High & $1(5.9)$ & $8(26.7)$ & $8(34.8)$ & \\
\hline Calcium supplement & $4(23.5)$ & 7 (23.3) & $11(47.8)$ & 0.118 \\
\hline Dose of L-thyroxine $(\mu \mathrm{g})$ & $136.8 \pm 28.5$ & $113.3 \pm 15.0$ & $110.9 \pm 18.6$ & $<0.001$ \\
\hline $\mathrm{TSH}(\mu \mathrm{lU} / \mathrm{mL})^{*}$ & $0.06(0.04-0.24)$ & $0.08(0.03-0.14)$ & $0.06(0.02-0.10)$ & 0.452 \\
\hline PTH (pg/mL) & $32.87 \pm 12.97$ & $33.15 \pm 13.71$ & $40.67 \pm 17.05$ & 0.134 \\
\hline $\mathrm{Ca}(\mathrm{mmol} / \mathrm{L})$ & $2.58 \pm 0.19$ & $2.46 \pm 0.14$ & $2.52 \pm 0.19$ & 0.071 \\
\hline 25-hydroxyvitamin D (ng/mL) & $15.37 \pm 6.41$ & $14.31 \pm 5.07$ & $13.68 \pm 6.37$ & 0.668 \\
\hline Number of BMD* & $2(2-2)$ & $2(2-2)$ & $2(2-2.5)$ & 0.621 \\
\hline Suppression time (month) ${ }^{*}$ & $6.3(5.9-8.2)$ & $7.1(5.9-9.5)$ & $7.2(5.8-10.7)$ & 0.544 \\
\hline Baseline BMD $\left(\mathrm{g} / \mathrm{cm}^{2}\right)$ & $0.998 \pm 0.127$ & $1.028 \pm 0.099$ & $0.896 \pm 0.111$ & $<0.001$ \\
\hline Last BMD $\left(\mathrm{g} / \mathrm{cm}^{2}\right)$ & $1.009 \pm 0.130$ & $1.041 \pm 0.119$ & $0.881 \pm 0.111$ & $<0.001$ \\
\hline
\end{tabular}

Results in the table: mean \pm SD/n (\%). *, median (Q1-Q3). BMI, body mass index; TSH, thyrotropin; FT3, free T3; FT4, free T4; PTH, parathyroid hormone; Ca, calcium; BMD, bone mineral density.

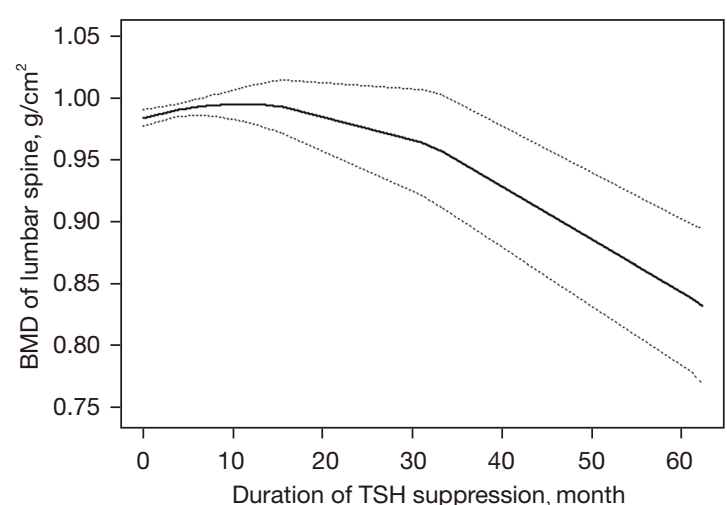

Figure 2 Curve fitting of lumbar BMD with a duration of TSH suppression (month) in all patients. After adjusting for age, BMI, risk of recurrence, calcium supplement, the dose of L-thyroxine, and the serum level of TSH, FT3, FT4, PTH, calcium, and 25-hydroxyvitamin D. BMD, bone mineral density; TSH, thyrotropin; BMI, body mass index; FT3, free T3; FT4, free T4; PTH, parathyroid hormone.

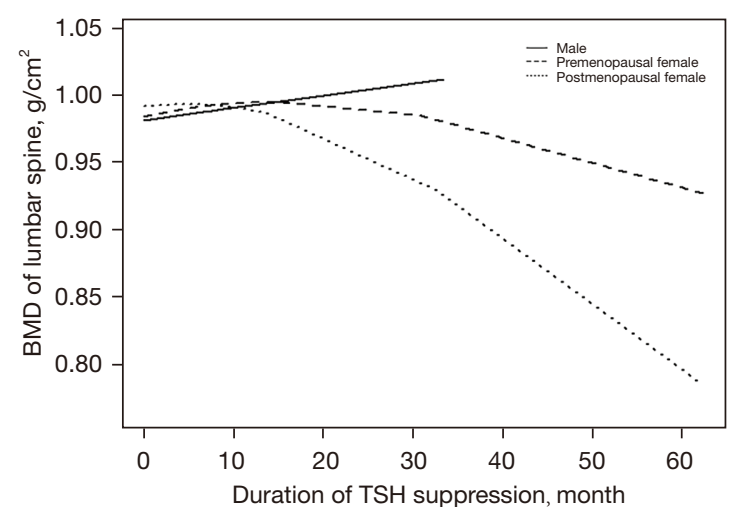

Figure 3 Curve fitting of lumbar BMD with a duration of TSH suppression (month) among the male, premenopausal, and postmenopausal women groups. After adjusting for age, BMI, risk of recurrence, calcium supplement, the dose of L-thyroxine, and the serum level of TSH, FT3, FT4, PTH, calcium, and 25-hydroxyvitamin D. BMD, bone mineral density; TSH, thyrotropin; BMI, body mass index; FT3, free T3; FT4, free T4; PTH, parathyroid hormone. 
Table 2 Comparison of general data between postmenopausal patients and postmenopausal controls

\begin{tabular}{|c|c|c|c|}
\hline Group & Premenopausal controls & Postmenopausal patients & $P$ value \\
\hline Age (years) & $62.0 \pm 9.4$ & $57.2 \pm 7.0$ & 0.058 \\
\hline BMI $\left(\mathrm{kg} / \mathrm{m}^{2}\right)$ & $23.3 \pm 3.6$ & $24.8 \pm 4.0$ & 0.193 \\
\hline Calcium supplement & $6(28.571)$ & $11(47.826)$ & 0.190 \\
\hline FT3 (pmol/L)* & $4.60(4.28-4.94)$ & $5.94(5.50-6.38)$ & $<0.001$ \\
\hline FT4 (pmol/L)* & $15.73(14.57-18.07)$ & $25.89(23.54-29.47)$ & $<0.001$ \\
\hline PTH (pg/mL) & $39.21 \pm 14.65$ & $40.67 \pm 17.05$ & 0.832 \\
\hline $\mathrm{Ca}(\mathrm{mmol} / \mathrm{L})$ & $2.50 \pm 0.17$ & $2.52 \pm 0.19$ & 0.809 \\
\hline Last BMD $\left(\mathrm{g} / \mathrm{cm}^{2}\right)$ & $0.822 \pm 0.110$ & $0.881 \pm 0.111$ & 0.083 \\
\hline
\end{tabular}

Results in the table: mean \pm SD/n (\%). *, median (Q1-Q3). BMI, body mass index; TSH, thyrotropin; FT3, free T3; FT4, free T4; PTH, parathyroid hormone; Ca, calcium; BMD, bone mineral density.

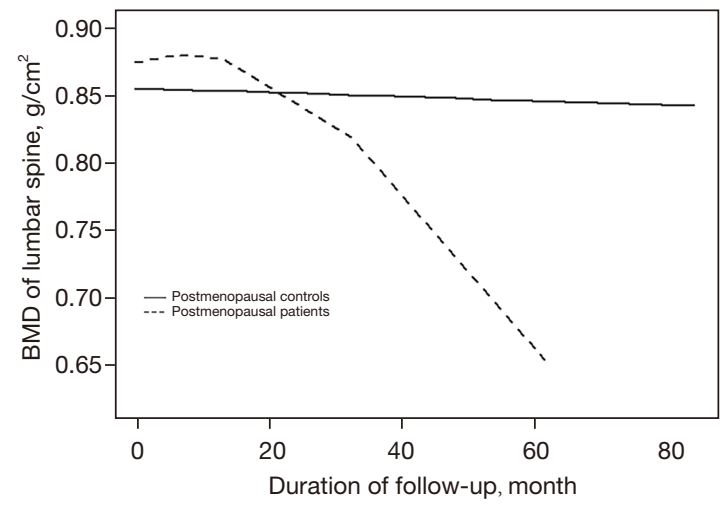

Figure 4 Curve fitting of lumbar BMD with a duration of follow-up (months) in postmenopausal patients and postmenopausal controls. After adjusting for factors of age, BMI, calcium supplement, and the serum level of TSH, FT3, FT4, PTH, and calcium. BMD, bone mineral density; BMI, body mass index; TSH, thyrotropin; FT3, free T3; FT4, free T4; PTH, parathyroid hormone.

month, which was statistically significant $(\mathrm{P}<0.001)$. The interaction between population grouping and calcium supplement was further analyzed, and the results showed that there was no interaction between the two factors
$(\mathrm{P}=0.244)$ (Figure 4)

\section{Changes of BMD with a duration of TSH suppression in different calcium supplement groups}

According to whether they took a calcium supplement or not, 70 patients were divided into the calcium supplement group (22 cases; $1,000 \mathrm{mg} /$ day) and the noncalcium supplement group (48 cases). In both the calcium supplement group and the non-supplement group, the BMD of the lumbar spine decreased significantly with the increase of suppression time (regression coefficient: -0.121 and $-0.147 ; \mathrm{P}=0.027$ and $\mathrm{P}<0.001$, respectively). After adjusting for the factors of age, BMI, population grouping, risk of recurrence, the dose of L-thyroxine, and the serum level of TSH, FT3, FT4, PTH, calcium, and 25-hydroxyvitamin $\mathrm{D}$, changes of BMD of the lumbar spine with time in different calcium supplementation groups had no statistically significant difference $(\mathrm{P}=0.534)$. We further analyzed the interaction between population grouping and calcium supplement intake, and the results showed that there was no interaction between the two factors $(\mathrm{P}>0.05)$ (Figure 5). 


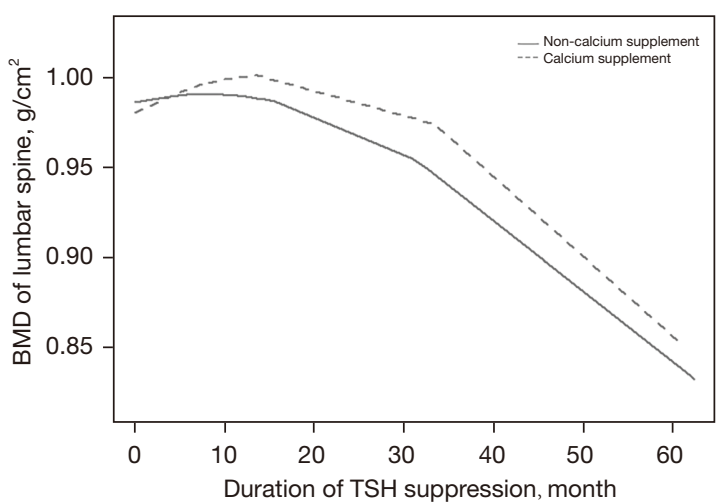

Figure 5 Curve fitting of lumbar BMD with a duration of TSH suppression (month) in different calcium supplement groups. Notes: After adjusting for factors of age, BMI, population grouping, risk of recurrence, the dose of L-thyroxine, and the serum level of TSH, FT3, FT4, PTH, calcium, and 25-hydroxyvitamin D. BMD, bone mineral density; TSH, thyrotropin; BMI, body mass index; FT3, free T3; FT4, free T4; PTH, parathyroid hormone.

\section{Discussion}

TSH can affect bone metabolism and BMD in various ways. TSH receptor (TSHR) exists in osteoblasts and osteoclasts. TSH can combine with TSHR to form a ligand-receptor complex, which directly affects bone reconstruction (27). TSH can also directly affect osteoclasts and osteoblasts. Takada et al. reported that TSH can suppress the formation and survival of osteoclasts through $\mathrm{JNK} / \mathrm{c}-$ jun and NF- $\mathrm{\kappa B}$ signaling pathways of RANK-L, thus affecting the function of osteoclasts. TSH affects the formation of osteoclasts by inhibiting tge TNF- $\alpha$ response and suppresses the differentiation of osteoclasts by downregulating Wnt and VEGF signals (28). TSH can affect osteoblasts by suppressing the expression of type 1 collagen in Runx-2 and transcription factors, thus inhibiting bone absorption and bone formation (29). All of these suggest the importance of TSH in bone metabolism. Subclinical hyperthyroidism caused by long-term TSH suppressive therapy will break the dynamic balance between osteoblasts and osteoclasts and will interfere with the process of bone metabolism (28). Furthermore, with the increase of treatment time, the bone density of some patients will be affected (30). This can partially explain our first conclusion. We found that the overall BMD value of all patients in the study decreased significantly with the increasing duration of TSH suppression, and this reduction was not affected by age and BMI.
Kim et al. [2015] followed up with 126 patients after a DTC operation. They found that TSH suppressive therapy accelerated bone loss, predominantly in postmenopausal women and exclusively during the early post-thyroidectomy period (14). Similarly, Kung et al. [1996] followed up with 46 postmenopausal DTC patients for 2 years and found that TSH suppressive therapy was associated with a bone mass reduction (17). Our results were similar to those of the above studies, suggesting that BMD is more likely to decrease in postmenopausal women with DTC after TSH suppressive therapy. The study of De Mingo Dominguez et al. [2018] showed that the trabecular bone fraction of postmenopausal women with DTC receiving TSH suppressive therapy was lower compared with that of premenopausal patients, suggesting that the bone microstructure was more prone to the deterioration (31). The study of Mohammadi et al. [2007] indicated that, in postmenopausal women with DTC, the bone mass decrease was more likely to occur in the early stage of TSH suppressive therapy (16). These conclusions suggest that we should intervene in postmenopausal women as early as possible. We should carry out osteoporosis education as soon as possible, increase the frequency of follow-up, and give anti-osteoporosis therapy as quickly as necessary.

We also found that, for the included patients in the study, a calcium supplement did not seem to improve BMD in the lumbar spine. As is well known, a calcium supplement is essential for the treatment of osteoporosis (32). In 1996, the study of Kung et al. [1996] showed that a calcium supplement could improve bone mass reduction in postmenopausal women who received TSH suppressive therapy after a DTC operation (17). Our results do not entirely agree with the above conclusions. However, there are differences between our experimental design and the research of Kung et al. [1996]. The main difference was that the patients included in our study were at intermediate or high risk of recurrence after total or subtotal DTC resection, whereas in the study of Kung et al. [1996], there was no strict requirement for risk stratification. Moreover, the mean age of patients in the study of Kung et al. [1996] was $63.4 \pm 7$ years, which was higher than the mean age of postmenopausal women in our study $(57.2 \pm 7$ years). In addition, only 23 postmenopausal women were included in our study. Among these 23 patients, 11 were supplemented with calcium, and 12 were not supplemented with calcium. Therefore, all of these factors may lead to different conclusions between our work and the study of Kung et al. [1996]. Our results suggest that, for the selected patients in 
our study, it may be not beneficial enough to take calcium supplements.

Our study had some limitations. We only studied the $\mathrm{BMD}$ of the lumbar spine, while the BMD data of the hip and the femoral neck were not included. Moreover, for osteoporosis, it would be more meaningful if some morphological markers of vertebral fractures were also added to the study. Also, the BMD measured by DXA is not a sensitive marker to indicate the change of bone strength. Some studies have shown that the BMD measured by DXA is relatively slow compared with the change of disease, and thus cannot reflect the trivial changes effectively and in a timely manner $(33,34)$. Furthermore, the number of patients in our group was limited. When we analyzed the subgroups, the number of patients in each subgroup was relatively small, which might affect the accuracy of the results. Finally, our median follow-up time would have benefited from being longer. It is possible that BMD changes in male and premenopausal females were not significant in short follow-up time.

\section{Conclusions}

The lumbar BMD of DTC patients decreased significantly after operation and ${ }^{131} \mathrm{I}$ therapy, especially in postmenopausal women. During the period of TSH suppression, calcium supplementation alone seems not to be sufficient enough to improve bone health estimated by lumbar BMD.

\section{Acknowledgments}

Funding: This study was supported by the Changzhou Science and Technology Program (No. CJ20210140).

\section{Footnote}

Reporting Checklist: The authors have completed the STROBE reporting checklist. Available at https:// gs.amegroups.com/article/view/10.21037/gs-22-50/rc

Data Sharing Statement: Available at https://gs.amegroups. com/article/view/10.21037/gs-22-50/dss

Conflicts of Interest: All authors have completed the ICMJE uniform disclosure form (available at https://gs.amegroups. com/article/view/10.21037/gs-22-50/coif). All authors report that this study was supported by the Changzhou Science and Technology Program (No. CJ20210140). The authors have no other conflicts of interest to declare.

Ethical Statement: The authors are accountable for all aspects of the work in ensuring that questions related to the accuracy or integrity of any part of the work are appropriately investigated and resolved. This study was conducted following the principles of the Declaration of Helsinki (as revised in 2013) and was approved by the ethics committee of the Third Affiliated Hospital of Soochow University [approval No. (2020) WD-018]. Written informed consent was obtained from the patients.

Open Access Statement: This is an Open Access article distributed in accordance with the Creative Commons Attribution-NonCommercial-NoDerivs 4.0 International License (CC BY-NC-ND 4.0), which permits the noncommercial replication and distribution of the article with the strict proviso that no changes or edits are made and the original work is properly cited (including links to both the formal publication through the relevant DOI and the license). See: https://creativecommons.org/licenses/by-nc-nd/4.0/.

\section{References}

1. Kitahara CM, Sosa JA. The changing incidence of thyroid cancer. Nat Rev Endocrinol 2016;12:646-53.

2. Haugen BR, Alexander EK, Bible KC, et al. 2015 American Thyroid Association Management Guidelines for Adult Patients with Thyroid Nodules and Differentiated Thyroid Cancer: The American Thyroid Association Guidelines Task Force on Thyroid Nodules and Differentiated Thyroid Cancer. Thyroid 2016;26:1-133.

3. Luster M, Aktolun C, Amendoeira I, et al. European Perspective on 2015 American Thyroid Association Management Guidelines for Adult Patients with Thyroid Nodules and Differentiated Thyroid Cancer: Proceedings of an Interactive International Symposium. Thyroid 2019;29:7-26.

4. Haugen BR, Sawka AM, Alexander EK, et al. American Thyroid Association Guidelines on the Management of Thyroid Nodules and Differentiated Thyroid Cancer Task Force Review and Recommendation on the Proposed Renaming of Encapsulated Follicular Variant Papillary Thyroid Carcinoma Without Invasion to Noninvasive Follicular Thyroid Neoplasm with Papillary-Like Nuclear Features. Thyroid 2017;27:481-3.

5. van der Wardt RA, Persoon AC, Klein Hesselink EN, et al. Long-Term Follow-Up for Differentiated Thyroid 
Carcinoma Patients: A Reconsideration. Thyroid 2017;27:475-6.

6. Kim TY, Kim WG, Kim WB, et al. Current status and future perspectives in differentiated thyroid cancer. Endocrinol Metab (Seoul) 2014;29:217-25.

7. Scheffel RS, Zanella AB, Dora JM, et al. Timing of Radioactive Iodine Administration Does Not Influence Outcomes in Patients with Differentiated Thyroid Carcinoma. Thyroid 2016;26:1623-9.

8. Lee SJ, Kim KM, Lee EY, et al. Low Normal TSH levels are Associated with Impaired BMD and Hip Geometry in the Elderly. Aging Dis 2016;7:734-43.

9. McGriff NJ, Csako G, Gourgiotis L, et al. Effects of thyroid hormone suppression therapy on adverse clinical outcomes in thyroid cancer. Ann Med 2002;34:554-64.

10. Delitala AP, Scuteri A, Doria C. Thyroid Hormone Diseases and Osteoporosis. J Clin Med 2020;9:1034.

11. Mosekilde L, Eriksen EF, Charles P. Effects of thyroid hormones on bone and mineral metabolism. Endocrinol Metab Clin North Am 1990;19:35-63.

12. Papaleontiou M, Hawley ST, Haymart MR. Effect of Thyrotropin Suppression Therapy on Bone in Thyroid Cancer Patients. Oncologist 2016;21:165-71.

13. Wang LY, Smith AW, Palmer FL, et al. Thyrotropin suppression increases the risk of osteoporosis without decreasing recurrence in ATA low- and intermediate-risk patients with differentiated thyroid carcinoma. Thyroid 2015;25:300-7.

14. Kim MK, Yun KJ, Kim MH, et al. The effects of thyrotropin-suppressing therapy on bone metabolism in patients with well-differentiated thyroid carcinoma. Bone 2015;71:101-5.

15. Mazokopakis EE, Starakis IK, Papadomanolaki MG, et al. Changes of bone mineral density in pre-menopausal women with differentiated thyroid cancer receiving L-thyroxine suppressive therapy. Curr Med Res Opin 2006;22:1369-73.

16. Mohammadi B, Haghpanah V, Tavangar SM, et al. Modeling the effect of levothyroxine therapy on bone mass density in postmenopausal women: a different approach leads to new inference. Theor Biol Med Model 2007;4:23.

17. Kung AW, Yeung SS. Prevention of bone loss induced by thyroxine suppressive therapy in postmenopausal women: the effect of calcium and calcitonin. J Clin Endocrinol Metab 1996;81:1232-6.

18. Zhang P, Xi H, Yan R. Effects of thyrotropin suppression on lumbar bone mineral density in postmenopausal women with differentiated thyroid carcinoma. Onco Targets Ther
2018;11:6687-92.

19. Jódar E, Begoña López M, García L, et al. Bone changes in pre- and postmenopausal women with thyroid cancer on levothyroxine therapy: evolution of axial and appendicular bone mass. Osteoporos Int 1998;8:311-6.

20. Kim CW, Hong S, Oh SH, et al. Change of Bone Mineral Density and Biochemical Markers of Bone Turnover in Patients on Suppressive Levothyroxine Therapy for Differentiated Thyroid Carcinoma. J Bone Metab 2015;22:135-41.

21. Sugitani I, Fujimoto Y. Effect of postoperative thyrotropin suppressive therapy on bone mineral density in patients with papillary thyroid carcinoma: a prospective controlled study. Surgery 2011;150:1250-7.

22. Vera L, Gay S, Campomenosi C, et al. Ten-year estimated risk of bone fracture in women with differentiated thyroid cancer under TSH-suppressive levothyroxine therapy. Endokrynol Pol 2016;67:350-8.

23. Yoon BH, Lee Y, Oh HJ, et al. Influence of Thyroidstimulating Hormone Suppression Therapy on Bone Mineral Density in Patients with Differentiated Thyroid Cancer: A Meta-analysis. J Bone Metab 2019;26:51-60.

24. Wang MY, Han ZQ, Gong XW, et al. TSH-suppressive therapy can reduce bone mineral density in patients with differentiated thyroid carcinoma: a meta-analysis. Eur Rev Med Pharmacol Sci 2020;24:922-9.

25. American Thyroid Association (ATA) Guidelines Taskforce on Thyroid Nodules and Differentiated Thyroid Cancer; Cooper DS, Doherty GM, et al. Revised American Thyroid Association management guidelines for patients with thyroid nodules and differentiated thyroid cancer. Thyroid 2009; 19:1167-214.

26. Wood SN. Low-rank scale-invariant tensor product smooths for generalized additive mixed models. Biometrics 2006;62:1025-36.

27. Kenkre JS, Bassett J. The bone remodelling cycle. Ann Clin Biochem 2018;55:308-27.

28. Takada I, Kouzmenko AP, Kato S. Wnt and PPARgamma signaling in osteoblastogenesis and adipogenesis. Nat Rev Rheumatol 2009;5:442-7.

29. Abe E, Marians RC, Yu W, et al. TSH is a negative regulator of skeletal remodeling. Cell 2003;115:151-62.

30. Biondi B, Cooper DS. Benefits of thyrotropin suppression versus the risks of adverse effects in differentiated thyroid cancer. Thyroid 2010;20:135-46.

31. De Mingo Dominguez ML, Guadalix Iglesias S, MartinArriscado Arroba C, et al. Low trabecular bone score in postmenopausal women with differentiated thyroid 
carcinoma after long-term TSH suppressive therapy. Endocrine 2018;62:166-73.

32. Drake MT, Clarke BL, Lewiecki EM. The Pathophysiology and Treatment of Osteoporosis. Clin Ther 2015;37:1837-50.

33. Yu EW, Thomas BJ, Brown JK, et al. Simulated increases in body fat and errors in bone mineral density

Cite this article as: Wang X, Teng R, Liu F, Liu P, Yang Y. Effect of thyrotropin suppressive therapy on lumbar bone mineral density in patients with differentiated thyroid cancer: a retrospective cohort study. Gland Surg 2022;11(2):432-441. doi: $10.21037 / g s-22-50$ measurements by DXA and QCT. J Bone Miner Res 2012;27:119-24.

34. Jain RK, Vokes T. Dual-energy X-ray Absorptiometry. J Clin Densitom 2017;20:291-303.

(English Language Editor: C. Mullens) 